\title{
Jogo bonito ou jogo feio: o que é e como podemos quantificar?
}

\section{Beautiful game or ugly game: what is it and how can we quantify it? Juego bello o juego feo: ¿qué es y cómo podemos cuantificarlo?}

\author{
Letícia Petruce Montoya $^{a}$ (D) , Júlia Barreirab* (iD) , Luciano Allegretti Mercadante ${ }^{a}$ (D)
}

Palavras-chave

Futebol;

Estética;

Indicadores de

desempenho.

\begin{abstract}
RESUMO
O objetivo desse estudo foi identificar quais variáveis quantitativas de uma partida de futebol são relacionadas à classificação de jogo bonito ou feio. A partir dos indicadores de desempenho disponíveis no site oficial da FIFA, selecionamos quatro jogos da Copa do Mundo de 2018 com características distintas. Os jogos foram assistidos por dez participantes que, em um questionário semiestruturado, apontaram os indicadores de desempenho que influenciaram o julgamento estético da partida. $O$ jogo bonito foi associado à grande quantidade de finalizações, chances de gols e passes certos. A ausência destes mesmos indicadores foi importante para a classificação dos jogos como feios. As variáveis quantitativas identificadas nesse estudo avançam com o debate sobre futebol e estética.
\end{abstract}

\section{Keywords}

Football;

Aesthetics;

Performance

indicators.

Palabras-clave

Fútbol;

Estética;

Indicadores de

rendimiento.

\begin{abstract}
The aim of this study was to identify the quantitative variables of a football match associated to the classification of a beautiful or ugly game. Based on the performance indicators 2018 FIFA World Cup, we selected four games with different characteristics. The games were watched by ten participants who pointed out the performance indicators that influenced the aesthetic judgment of the game. A semi-structured questionnaire was used for data collection. The beautiful game was associated with a large number of shots, shots on goal and pass accuracy. The absence of these same indicators was important for classifying the games as ugly. The quantitative variables identified in this study advance the debate on football and aesthetics.
\end{abstract}

\section{RESUMEN}

El objetivo de este estudio fue identificar qué variables cuantitativas de un partido de fútbol están relacionadas con la clasificación de un juego bello o feo. Hemos seleccionado cuatro juegos de la Copa Mundial 2018 con diferentes características de los indicadores de rendimiento. Los juegos fueron observados por diez voluntarios que, en un cuestionario semiestructurado, señalaron los indicadores de rendimiento que influyeron en el juicio estético del juego. El juego bello se asoció con la gran cantidad de presentaciones, posibilidades de goles y pases ciertos. La ausencia de estos mismos indicadores fue importante para clasificar los juegos como feos. Las variables cuantitativas identificadas en este estudio avanzan el debate sobre el fútbol y la estética. 


\section{INTRODUÇÃO}

O futebol é o esporte de maior alcance mundial, sendo praticado e assistido por homens e mulheres de diferentes nacionalidades (Stolen et al., 2005). A Copa do Mundo corresponde à principal competição mundial da modalidade e, durante o evento, torcedores costumam se reunir para assistirem os jogos e torcerem por suas seleções. No decorrer da partida, é comum que questões relacionadas às características e aos julgamentos estéticos dos jogos sejam evocadas e que os conceitos de bonito e feio, bom e ruim, sejam utilizados nessas classificações (Borge et al., 2015). Também encontramos o uso frequente da expressão "jogo bonito" na literatura científica sobre futebol (Conn, 2010; Dubal, 2010; Hallinan e Hughson, 2009; Taylor, 1998; Vrooman, 2007). Apesar da popularidade das expressões, as definições ainda são desconhecidas e apresentam potencial para serem exploradas cientificamente.

O conceito de "beleza" e sua relação com o esporte já foram explorados em diversos estudos da filosofia, principalmente na área da estética (Arnold, 1990; Best, 1974; Edgar, 2013). Os autores sugerem que a relação entre estética e esporte é mediada pelo jogo. No jogo, o ser humano age, interage, encena, se comunica, se conhece, cria, reconstrói, se reinventa e redescobre (Lacerda, 2002b). Essa dinâmica permite uma experiência imersa na dimensão da arte e da estética. Apesar da dificuldade em definir o conceito, a estética está usualmente relacionada à capacidade de sensorialidade e sensibilidade de apreciar ou julgar algo (Lacerda, 2002a). Essa ação é realizada por um livre interesse, sendo motivada pela atração e pelo prazer da experiência (Damo, 2001; Gumbrecht, 2001).

Entre os estudiosos da área, não há um consenso em relação à extensão da estética e às características de uma experiência estética no futebol (Borge et al., 2015). Nesse sentido, diversas variáveis de uma partida poderiam ser exploradas buscando identificar aquelas que influenciam a percepção do espectador. Um aspecto frequentemente debatido na associação entre futebol e estética é a relação entre o jogar bem e o jogar bonito, isto é, entre o desempenho das equipes e a estética da partida. De acordo com Graça e Lacerda (2011), existe uma dependência entre o belo e o bom que pode ser extrapolada para uma aliança entre estética e desempenho. É possível que indicadores de desempenho utilizados em partidas de futebol, como o resultado final do jogo, o número de gols e a quantidade de finalizações, estejam relacionados com o julgamento estético dos torcedores (Hughes e Bartlett, 2002; Lago-Ballesteros e Lago-Peñas, 2010). Algumas dessas relações já foram investigadas por estudos anteriores e serão exploradas abaixo mostrando como o presente estudo pode avançar com o conhecimento já existente.

A relação entre resultado final e a estética do jogo ainda é contraditória na literatura científica. Por exemplo, para Gumbrecht (2001), a experiência estética é desvinculada do resultado final. Segundo o autor, aproximação entre estética e esporte depende da competência dos torcedores em julgarem se um jogo foi bonito ou feio, independente do placar final. Nesse sentido, um torcedor é capaz de classificar um jogo como bonito, mesmo que seu time não seja o vencedor do confronto. Já para Damo (2001), essa capacidade de dissociação entre resultado e beleza é um desafio, à medida que as excitações geradas pelo pertencimento clubístico, assim como pela aversão ao adversário, podem influenciar a percepção do torcedor.

Em relação às finalizações e à quantidade de gols, Graça e Lacerda (2011) sugerem que essas variáveis são centrais na construção de jogo, pois conferem sentido e significado a ele. Sabemos que o jogo de futebol é caracterizado por muitas finalizações e poucos gols, tornando o gol um aspecto essencial na estética desta modalidade (Graça e Lacerda, 2011). Entretanto, jogos com uma baixa competitividade entre as equipes podem reverter essa lógica e apresentar uma elevada quantidade de gols, minimizando as expectativas do torcedor em relação ao acontecimento do gol e a diminuindo euforia produzida pela imprevisibilidade do mesmo (Barreira e Silva, 2016). Nesse sentido, podemos avançar com o conhecimento ao investigar se há uma relação diretamente proporcional entre número de gols e experiência estética, ou se esta associação apresenta um ponto de saturação, mediada pela competitividade da partida.

A posse de bola é considerada um elemento central no sucesso do futebol moderno, mas sua relação com o desempenho esportivo ainda é contraditória (Collet, 2013). Comumente utilizada para indicar a superioridade de um time, estudos mostram que equipes que estão perdendo um jogo tendem a manter a posse de bola para avançar no campo adversário e criar mais chances de finalizações (Collet, 2013; Jones et al., 2004; LagoPeñas e Dellal, 2010; Lago, 2009). Desta forma, a posse de bola parece estar mais relacionada à estética do jogo do que ao próprio desempenho das equipes e pode ser explorada cientificamente (Bate, 1987).

Outro aspecto que ainda está aberto para investigação é a associação entre esporte, estética e ética (Borge et al., 2015). Diversas questões éticas podem emergir em uma partida de futebol, sendo a agressão física a mais frequente. Para Gumbrecht (2001) o futebol é considerado como um esporte "violento" dado que suas regras permitem o contato entre os jogadores e que esses sejam alvo de agressão. As variáveis de número de faltas, quantidade de cartões amarelos e vermelhos geram informações sobre contato físico durante o jogo. Nesse sentido, essas variáveis também podem ser exploradas em relação à percepção dos espectadores, verificando se exercem influência no julgamento estético da partida.

Apesar das diversas relações ainda abertas e que podem ser investigadas entre estética e esporte, a associação entre beleza e imprevisibilidade do jogo 
parece consolidada entre os estudiosos (Damo, 2001; Gumbrecht, 2001). Ao assistir a um jogo, os torcedores anseiam por belas jogadas sem a certeza de que elas acontecerão (Gumbrecht, 2001). A imprevisibilidade é ainda mais marcante ao assumir que a bela jogada jamais foi vista anteriormente pela maioria dos espectadores e que, em muitos casos, ela de fato está sendo posta em ação desse modo pela primeira vez (Gumbrecht, 2001). Diferentemente das demais variáveis apresentadas anteriormente, a imprevisibilidade não pode ser quantificada, mas se constitui como um elemento central na estética da partida.

Como mostrado acima, a literatura científica apresenta as contradições ainda existentes e associações já consolidadas entre as características de uma partida e os julgamentos estéticos dos torcedores. O presente estudo pretende avançar com o debate ao investigar quais indicadores de desempenho são relacionados aos conceitos de jogo bonito ou feio. Portanto, o objetivo desse estudo foi identificar quais variáveis quantitativas de uma partida de futebol são relacionadas à classificação de jogo bonito ou feio.

\section{MÉTODOS}

Nesse estudo utilizamos o método misto (Creswell \& Plano Clark, 2011). Esse método é caracterizado por uma primeira etapa quantitativa seguida por uma etapa qualitativa. Na primeira etapa, realizamos o levantamento dos indicadores de desempenho de todos os jogos da Copa do Mundo de Futebol Masculino 2018 disponíveis no website oficial da FIFA. Em seguida, as variáveis coletadas foram exploradas a partir de ferramentas estatísticas, com o objetivo de identificar jogos com características distintas. Na segunda etapa, oito jogos foram assistidos pelos pesquisadores e quatro deles foram escolhidos para compor a amostra final desse estudo. Os jogos selecionados foram assistidos pelos voluntários e classificados entre jogo bonito ou feio. Para essa classificação, utilizamos um questionário semiestruturado que permitiu identificar quais variáveis foram associadas à estética da partida. Por fim, os indicadores quantitativos e as opiniões dos voluntários foram confrontados para responder o objetivo do estudo. Todas as etapas do método desse estudo são apresentas de forma resumida na Figura 1, e descritas detalhadamente a seguir.

\section{SELEÇÃO DAS PARTIDAS}

Para minimizar a influência do viés do torcedor sobre o julgamento estético, utilizamos os jogos da Copa do Mundo de Futebol Masculino de 2018. Com o objetivo de identificar partidas com características distintas, coletamos os indicadores de desempenho de ambas as equipes em todos os jogos da primeira fase da competição. Apenas os jogos das fases de grupo foram considerados nesse estudo, com o objetivo de evitar que os voluntários se lembrassem dos placares e das dinâmicas, normalmente mais marcantes nas fases finais do campeonato. Todos os dados foram coletados no website oficial da FIFA.

A partir do banco de dados coletado, composto por 48 jogos, selecionamos oito partidas. Desconsideramos as partidas disputadas pela seleção brasileira para evitar a influência do pertencimento clubístico no julgamento estético dos voluntários. Nessa etapa, utilizamos a estatística descritiva, com medidas de dispersão e posição, gráficos e tabelas, para explorarmos o banco de dados e identificarmos os jogos com características distintas. Após a seleção, os pesquisadores assistiram os oito jogos garantindo que os indicadores de desempenho também fossem percebidos pelos espectadores durante a partida. A partir dos dados quantitativos e das análises

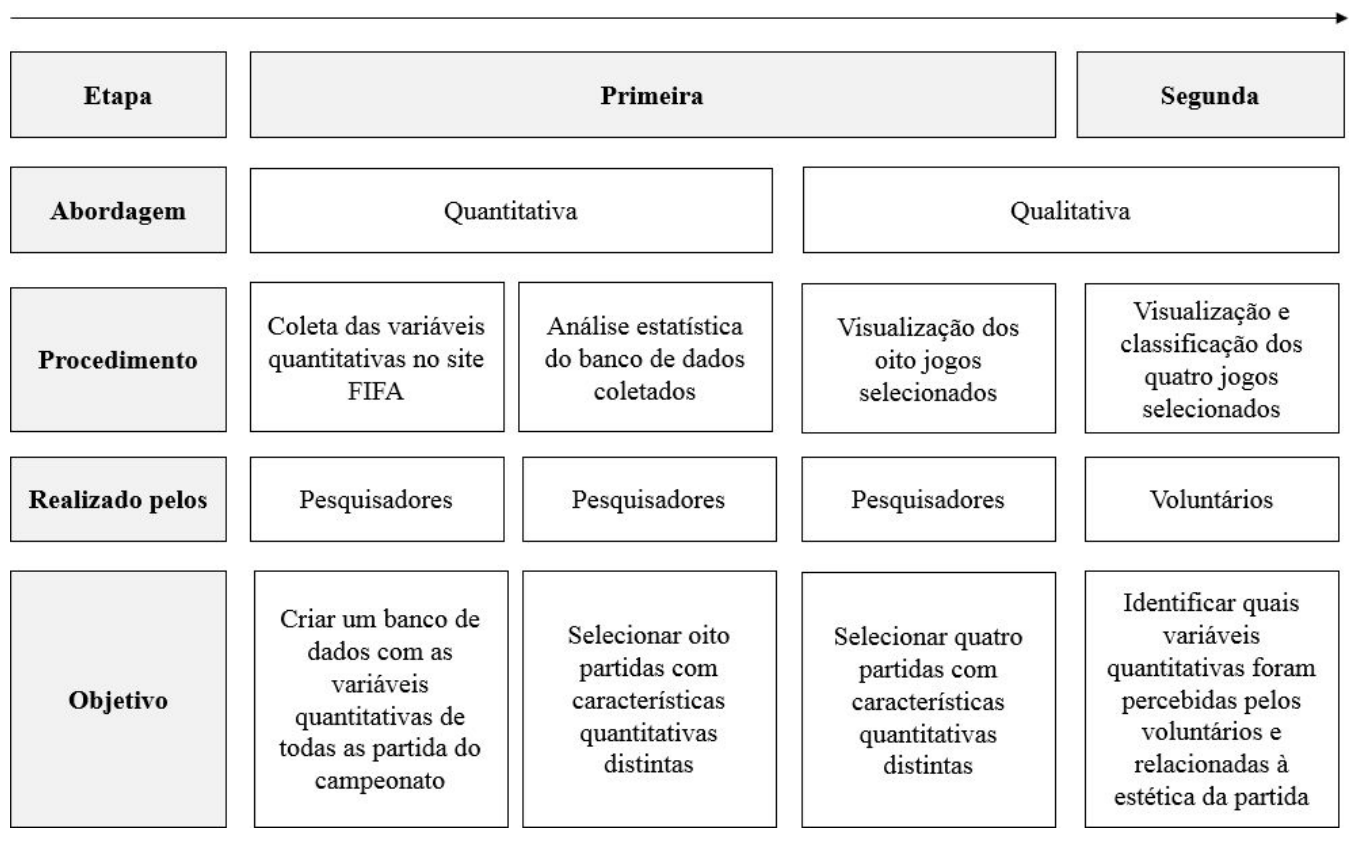

Figura 1. Método misto utilizado no presente estudo. 
dos jogos, quatro deles compuseram a amostra final desse estudo. A Tabela 1 apresenta os quatros jogos selecionados e os indicadores de desempenho de cada equipe.

Para a seleção dos jogos, nos baseamos na revisão da literatura e na possível relação de alguns indicadores de desempenho com a estética da partida. O Jogo 1 foi escolhido devido ao próximo número de gols entre as equipes e à inversão do time vencedor e perdedor ao longo da partida. Além disso, o jogo 1 apresenta uma grande quantidade de faltas. O Jogo 2 foi escolhido pela grande diferença quanto ao tempo de posse de bola e pelo alto número de finalizações com baixa quantidade de gols. Além disso, os gols foram feitos nos acréscimos do segundo tempo. O Jogo 3 foi selecionado principalmente pelo grande número de finalizações e gols na partida. A grande diferença no tempo de posse de bola entre as equipes também foi utilizada como critério para escolha. Por fim, o Jogo 4 foi escolhido pela grande diferença no tempo de posse de bola entre as equipes, e por ser uma partida com poucas finalizações e gols.

\section{PARTICIPANTES}

Os jogos selecionados foram assistidos por dez participantes selecionados por um processo de amostra por conveniência (Peterson e Merunka, 2014). Como critérios de inclusão, poderiam participar desse estudo indivíduos maiores de 18 anos e devidamente matriculados nos cursos de graduação ou pós-graduação da Faculdade de Ciências Aplicadas (FCA) da UNICAMP, local onde foi realizada a coleta de dados. Além disso, os participantes deveriam ter cinco dias disponíveis para a realização do estudo e concordar em assinar o Termo de Consentimento Livre e Esclarecido. O presente projeto foi aprovado pelo Comitê de Ética e Pesquisa da UNICAMP (CAAE: 82887118.3.0000.5404).

Também utilizamos como critério de inclusão a experiência dos participantes com a modalidade de futebol. Para participar do estudo, os indivíduos deveriam praticar e/ou atuar como treinadores de futebol por pelo menos 10 anos. De acordo com Damo (2001), o prazer estético depende do entendimento da dinâmica do jogo. Por esse motivo, puderam participar do estudo somente indivíduos que conheçam a dinâmica interna da partida de futebol. Devido à imprevistos, nem todos os participantes puderam participar da análise de todos os jogos, ainda assim, mais da metade dos voluntários esteve presente encontro.

As características dos participantes são apresentadas na Tabela 2.

\section{PROCEDIMENTOS}

A coleta de dados ocorreu em cinco encontros realizados na FCA/UNICAMP em Limeira. No primeiro encontro, foram explicados os objetivos do estudo, assim como o método de coleta de dados. Nos quatro encontros seguintes os participantes assistiram aos jogos selecionados e, ao final, responderam o questionário estruturado que permitiu a caracterização de cada partida. Os jogos foram assistidos em conjunto para

Tabela 1. Indicadores de desempenho das partidas selecionadas para análise.

\begin{tabular}{|c|c|c|c|c|c|c|c|c|}
\hline & \multicolumn{2}{|c|}{ Jogo 1} & \multicolumn{2}{|c|}{ Jogo 2} & \multicolumn{2}{|l|}{ Jogo 3} & \multicolumn{2}{|c|}{ Jogo 4} \\
\hline & Sérvia & Suíça & Coreia & Alemanha & Bélgica & Tunísia & Irã & Espanha \\
\hline Gols & 1 & 2 & 2 & 0 & 5 & 2 & 0 & 1 \\
\hline Finalizações & 12 & 20 & 11 & 26 & 23 & 15 & 5 & 17 \\
\hline Finalizações no alvo & 3 & 5 & 5 & 6 & 12 & 5 & 0 & 3 \\
\hline Finalizações fora do alvo & 7 & 8 & 5 & 11 & 8 & 6 & 5 & 6 \\
\hline Defesas & 2 & 7 & 1 & 9 & 3 & 4 & 0 & 8 \\
\hline Finalizações no trave & 0 & 1 & 0 & 0 & 1 & 0 & 0 & 0 \\
\hline Escanteios & 3 & 7 & 3 & 9 & 5 & 2 & 2 & 6 \\
\hline Impedimentos & 0 & 3 & 0 & 1 & 3 & 3 & 2 & 2 \\
\hline Posse de bola (\%) & 42 & 58 & 30 & 70 & 52 & 48 & 30 & 70 \\
\hline Precisão de passe (\%) & 78 & 87 & 74 & 88 & 84 & 82 & 69 & 89 \\
\hline Passes & 309 & 547 & 237 & 719 & 468 & 477 & 219 & 805 \\
\hline Passes completos & 241 & 474 & 176 & 633 & 391 & 392 & 152 & 718 \\
\hline Distância percorrida $(\mathrm{km})$ & 116 & 112 & 118 & 115 & 104 & 102 & 106 & 105 \\
\hline Bolas recuperadas & 44 & 40 & 43 & 39 & 44 & 37 & 50 & 35 \\
\hline Carrinhos & 11 & 14 & 10 & 9 & 4 & 10 & 17 & 5 \\
\hline Cartões amarelos & 4 & 1 & 4 & 0 & 0 & 1 & 2 & 0 \\
\hline Cartões vermelhos & 0 & 0 & 0 & 0 & 0 & 0 & 0 & 0 \\
\hline Faltas & 17 & 12 & 16 & 7 & 12 & 13 & 14 & 14 \\
\hline Tempo dos gols & $5^{\prime}$ & $52^{\prime}-90^{\prime}$ & $90^{\prime}+3^{\prime}-90^{\prime}+6^{\prime}$ & - & 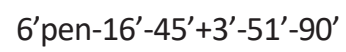 & $18^{\prime}-90^{\prime}+3^{\prime}$ & - & $54^{\prime}$ \\
\hline Pênaltis & 0 & 0 & 0 & 0 & 1 & 0 & 0 & 0 \\
\hline
\end{tabular}


Tabela 2. Características dos participantes do estudo.

\begin{tabular}{ccc}
\hline Característica & Categorias & Frequência (\%) \\
\hline Gênero & Mulheres & $4(40 \%)$ \\
& Homens & $6(60 \%)$ \\
Escolaridade & Graduação & $6(60 \%)$ \\
& Pós-graduação & $4(40 \%)$ \\
\hline
\end{tabular}

Legenda: Dados expressos em frequência absoluta (e relativa).

simular a situação real em que torcedores se reúnem para assistir futebol, assim como trocam opiniões e comentários durante a transmissão. Cada encontro teve duração de aproximadamente duas horas.

\section{INSTRUMENTO}

Para coleta de dados, elaboramos um questionário aberto, que incluía, logo no início, uma pequena caracterização dos participantes, com o nome, idade, há quanto tempo praticavam futebol e se já tinham atuado na área. Após essa caracterização, pedimos para que eles classificassem o jogo assistido como bonito ou feio. Por fim, pedimos que apontassem quais características do jogo os levaram a essa conclusão e que apresentassem suas impressões sobre o jogo. Dessa forma, foi possível identificar quais foram os indicadores quantitativos mais percebidos pelos participantes, e como eles relacionaram às classificações de jogo bonito ou feio.

\section{ANÁLISE FINAL}

Todos os questionários foram lidos, transcritos e analisados pelos pesquisadores. Para cada jogo agrupamos as classificações de bonito ou feio e buscamos identificar similaridades entre as opiniões dos participantes. Nesse processo, utilizamos a Análise de Conteúdo definida como a identificação do conteúdo presente nas mensagens que permita estabelecer relação com conceitos relativos à determinado tema de pesquisa (Creswell e Plano Clark, 2011).

O procedimento básico da análise de conteúdo se refere à definição de categorias e subcategorias pertinentes aos propósitos da pesquisa. As categorias e subcategorias podem ser construídas de três formas: fechada, aberta ou mista. A forma fechada envolve uma abordagem dedutiva, ou seja, as categorias são pré-escolhidas a partir da literatura existente. Nesse estudo, utilizamos as variáveis apresentadas na revisão de literatura e relacionadas por estudos anteriores com a estética da partida para construir as categorias. $\mathrm{Na}$ forma aberta, as categorias são construídas durante o andamento da pesquisa, o que também é conhecido como abordagem indutiva, na qual os resultados obtidos geram novos conceitos e teorias. Caso novos elementos aparecessem durante as leituras, novas categorias podem ser criadas. Portanto, nesse estudo utilizamos a forma mista para a análise, dado que as categoriais foram construídas de forma fechada e aberta, caracterizando a abordagem dedutiva-indutiva.
Este último tipo citado, permite adaptações que proporcionam novas contribuições à modelos teóricos existentes (Krippendorff, 2013). Dessa forma, foi possível identificar quais indicadores de desempenho foram relacionados às classificações de jogo bonito ou feio, realizadas pelos voluntários do estudo.

\section{RESULTADOS}

A Tabela 3 apresenta as variáveis utilizadas pelos participantes nas classificações de cada jogo, classificado como bonito ou feio. Os participantes do estudo apresentaram opiniões contraditórias em relação ao jogo 1. A partida foi considerada como bonita devido à "quantidade de chances claras de gols; número de gols em uma partida; decisão do resultado da partida acontecer nos últimos minutos" (Participante 1). Além disto, alguns participantes relataram aspectos táticos como fatores determinantes para a classificação estética do jogo. Por exemplo, o jogo foi considerado como bonito devido à "defesa na maioria do tempo em bloco, alta e com pressão na bola; jogo ofensivo rápido e busca de profundidade" (Participante 2). Em contrapartida, o Participante 6 apresentou uma opinião oposta, como mostrada abaixo:

Classifico esse jogo como um jogo relativamente feio; muitos erros de passe; muita falta desnecessária no jogo; diversos "chutões" sem pensar no jogo; muitas bolas forçadas e poucas bolas realmente trabalhadas; muito chute a gol que não apresentou real perigo aos goleiros. (Participante 6).

Notamos que as chances de gols apresentaram influência na estética do jogo 1 , tanto positiva quanto negativamente. Alguns participantes perceberam muitas chances, enquanto outros perceberam poucas, e ambas influenciaram o julgamento estético.

Encontramos um maior consenso entre os participantes na classificação dos jogos feios. $O$ jogo 2 foi considerado feio devido às "pouquíssimas chances de gol e muitos erros de passe" (Participante 5). Essa opinião foi corroborada por outro participante, que afirmou: "os jogadores estavam sem vontade de jogar, não houve lances de perigo e emoção" (Participante 8). As características táticas também foram importantes na classificação do jogo considerado como feio, por ser "excessivamente de transição de ambas as equipes" (Participante 4). 
Tabela 3. Opinião dos participantes em relação à estética de cada jogo.

\begin{tabular}{|c|c|c|}
\hline & Bonito & Feio \\
\hline Jogo 1 & - Muitas chances de gols ( 2 ) & - Poucas chances de gols ( 2 ) \\
\hline$(n=9)$ & · Decisão nos momentos finais do jogo (2) & - Muitos passes errados (2) \\
\hline Jogo 2 & & · Poucas chances de gols (4) \\
\hline \multirow[t]{3}{*}{$(n=6)$} & & - Muitos passes errados (2) \\
\hline & & - Poucas finalizações (2) \\
\hline & & - Grande diferença na posse de bola (1) \\
\hline Jogo 3 & - Muitos passes certos (2) & \\
\hline \multirow[t]{2}{*}{$(n=7)$} & · Elevado número de gols (3) & - Muitos passes errados (2) \\
\hline & · Muitas finalizações (4) & \\
\hline Jogo 4 & & - Muitos passes errados (2) \\
\hline \multirow[t]{4}{*}{$(n=8)$} & & - Poucas finalizações (2) \\
\hline & & - Pouca chance de gol (2) \\
\hline & & - Grande diferença na posse de bola (1) \\
\hline & & · Muitos chutões (2) \\
\hline
\end{tabular}

Legenda: O número entre parênteses representa a quantidade de indivíduos que expressaram determinada opinião. $n=$ quantidade de voluntários que assistiram e analisaram o jogo.

O jogo 4 apresentou o mesmo padrão, sendo classificado por todos os voluntários como feio. De acordo com o Participante 3, o jogo apresentou "muitos chutões e ataques desperdiçados; domínio da Espanha sem resultados em gols e efetividade de chutões". A opinião foi reforçada por outro participante, ao apontar que: "não houve muitas assistências, pouca competitividade, poucas chances reais de gol; jogo muito truncado" (Participante 9).

O terceiro jogo foi considerado pela maioria dos voluntários como um jogo bonito, principalmente pelo elevado número de gols e finalizações. Essa percepção é expressa na fala abaixo:

Considero esse jogo bonito. Achei que mesmo a Tunísia não ter conseguido sai jogando direito e a Bélgica ter ficado no controle do jogo a todo momento, o jogo foi bonito pela quantidade de oportunidades criadas, ou seja, a todo momento tinha algo interessante acontecendo, tanto é que saíram muitos gols. (Participante 7).

Entretanto, a variável de passes apresentou contradições na percepção dos voluntários. Para alguns, os passes errados apareceram como prejudicial à estética do jogo, já para outros, a quantidade de passes certos foi um elemento importante para classificar o jogo como bonito. Nenhum dos participantes fez menção às faltas do jogo ou à quantidade de cartões vermelhos e amarelos no julgamento estético da partida.

\section{DISCUSSÃO}

Os resultados do estudo são interessantes, tanto no contexto científico quanto no prático. O futebol, além de fazer parte da cultura brasileira, é o esporte mais popular do mundo e desperta grande curiosidade por parte da sociedade. Ao analisar a estética do jogo de futebol, assunto normalmente abordado por estudiosos da filosofia, nosso estudo possibilitou avançar com o debate ao trazer dados quantitativos para um melhor entendimento do fenômeno. Identificamos que os passes, as finalizações e as chances de gols são as principais variáveis associadas à estética da partida de futebol.

Encontramos um maior consenso entre os participantes na classificação dos jogos como feios. As poucas finalizações, poucas chances de gols, grande diferença na posse de bola e grande quantidade de passes errados foram as principais variáveis quantitativas utilizadas para esta classificação. A análise quantitativa realizada na primeira etapa do estudo corrobora a opinião dos voluntários, ao mostrar que os jogos 2 e 4, classificados como feios, apresentam um baixo número de finalizações e uma grande diferença no tempo de posse de bola entre as equipes em relação aos demais jogos da Copa do Mundo. As chances de gols e os passes errados apareceram como novas variáveis na caracterização das partidas.

As finalizações e chances de gols também apareceram como variáveis importantes na classificação dos jogos bonitos. Além da sua relação do desempenho das equipes (Lago-Ballesteros e Lago-Peñas, 2010), essas variáveis aparecem como centrais na apreciação estética. As finalizações e as chances de gol geram expectativas e emoções no espectador. A imprevisibilidade e a tensão gerada com a possibilidade do gol influenciam positivamente a experiência de quem está assistindo ao jogo e, em contrapartida, a ausência desses sentimentos parece importante no julgamento negativo.

Da mesma forma, os passes (certos e errados) também aparecerem como uma variável que influencia tanto positivamente quanto negativamente a estática do jogo de futebol. A posse de bola foi apontada pelos 
participantes apenas na classificação dos jogos como feios. Estas variáveis estão relacionadas com a fluidez e a dinamicidade do jogo. Os resultados desse estudo mostram que um jogo dinâmico e fluido, ou seja, com poucos erros de passe e a manutenção de posse de bola distribuída por ambas as equipes, são favoráveis para a apreciação estética. Além disso, estudos anteriores mostraram que as finalizações e os gols acontecem normalmente em sequência de passes mais longas (Barreira et al., 2016; Hughes e Franks, 2005). Portanto, a relação entre passes certos, finalizações e desempenho também pode ter favorecido a classificação estética do jogo.

Apesar da falta de consenso, o jogo 3 foi considerado como bonito pela maioria dos participantes. Entre todos os jogos selecionados para o estudo, este jogo apresentou o maior número de gols, variável também apontada pelos voluntários como importante na classificação do jogo como bonito. Esse resultado reforça que o gol representa um aspecto essencial na estética do jogo de futebol (Graça e Lacerda, 2011). Estudos futuros podem avançar com o conhecimento, ao explorar a relação entre gol e estética de uma maneira mais robusta.

Nenhum participante apontou as faltas como uma variável importante na apreciação estética do jogo de futebol. Encontramos uma média de 26 faltas nos jogos analisados nesse estudo. Nacionalmente, Chiminazzo et al. (2013) encontraram uma média de 41 faltas nos jogos do Campeonato Brasileiro. Possivelmente, os participantes perceberam uma baixa quantidade de faltas nas partidas da Copa do Mundo, que compuseram a amostra desse estudo, em relação aos jogos dos campeonatos nacionais, comumente assistidos por torcedores brasileiros.

Os aspectos táticos também apareceram no julgamento estético do jogo de futebol. Damo (2001) sugere que o conhecimento sobre a dinâmica do jogo é fundamental para a apreciação estética. Nesse sentido, utilizamos a atuação como jogador ou treinador de futebol por pelo menos dez anos como critério de seleção dos voluntários para participar do estudo. Todos os participantes eram graduandos ou pós-graduandos nos cursos de Ciências do Esporte ou Educação Física e a maioria já atuou como treinador de futebol. Estas características da amostra podem explicar o a razão dos aspectos táticos também serem apontados pelos participantes e vinculados à classificação estética do jogo.

\section{CONSIDERAÇÕES FINAIS}

A análise das partidas da Copa do Mundo de futebol masculino mostrou que os passes, as finalizações e as chances de gols são as principais variáveis quantitativas associadas à estética do jogo de futebol. $\mathrm{O}$ jogo bonito está associado à grande quantidade de finalizações, chances de gols e passes certos. As poucas finalizações, poucas chances de gols, grande diferença na posse de bola e grande quantidade de passes errados foram as principais variáveis quantitativas utilizadas para os jogos como feios. Também notamos um maior consenso entre os voluntários na classificação dos jogos como feios. Por fim, as faltas não foram apontadas como uma variável importante na apreciação estética da partida.

Apesar de trazer novas discussões e achados sobre a relação entre estética e futebol, é importante reconhecer as limitações desse estudo. A maior limitação é em relação ao processo de amostragem utilizado. Os resultados poderiam ser ainda mais confiáveis e passíveis de generalização com maior número de participantes, assim como se eles apresentassem características mais heterogêneas. Além disso, os dados quantitativos utilizados para a seleção dos jogos foram extraídos do site da FIFA e sua confiabilidade não foi analisada. Por fim, os aspectos táticos citados pelos participantes não foram explorados porque não eram o objeto de estudo, mas apresentam potencial para serem analisados por investigações futuras.

\section{FINANCIAMENTO}

O presente trabalho contou com apoio financeiro do Conselho Nacional de Desenvolvimento Científico e Tecnológico (CNPq).

\section{CONFLITOS DE INTERESSE}

Os autores declaram não haver conflitos de interesse.

\section{REFERÊNCIAS}

Arnold PJ. Sport, the aesthetic and art: further thoughts. Br J Educ Stud 1990;38(2):160-79. http://dx.doi.org/10.1080 /00071005.1990.9973844.

Barreira J, Silva CE. National teams in Women's Soccer World Cup from 1991 to 2015: participation, performance and competitiveness. J Phys Educ Sport 2016;16(3):795-9.

Barreira J, Vendite C, Vendite LL. Analysis of shots and passing sequence of a soccer team and its opponents during 2014 Brazilian championship. Int J Sports Sci 2016;6(4):163-7.

Bate R. Football chance: tactics and strategy. In Reilly T, Lees A, Davids K, Murphy WJ, editors. Science and Football: Proceedings of the first World Congress of Science and Football; 1987; Liverpool. Liverpool: Routledge; 1987.

Best D. The aesthetic in sport. Br J Aesthet 1974;14(3):197-213. http://dx.doi.org/10.1093/bjaesthetics/14.3.197.

Borge S, Smith M, Bruun Vaage M. The aesthetics of football. Sport Ethics Philos 2015;9(2):93-6. http://dx.doi.org/10. 1080/17511321.2015.1048822.

Chiminazzo JGC, Mascara DI, Del Vecchio FB. Estudo descritivo da distribuição de gols, faltas e cartões no Campeonato Paulista 2008-Série A1. Rev Bras Futsal Fut 2013;5(15):80-7.

Collet $\mathrm{C}$. The possession game? A comparative analysis of ball retention and team success in European and international football, 2007-2010. J Sports Sci 2013;31(2):123-36. http://dx.doi.org/10.1080/02640414.2012.727455. PMid:23067001. 
Conn D. The beautiful game? Searching for the soul of football. New York: Random House; 2010.

Creswell JW, Plano Clark VL. Designing and conducting mixed methods research (2nd ed.). Los Angeles: SAGE Publications; 2011.

Damo AS. Futebol e estética. São Paulo Perspect 2001;15(3):82-91. http://dx.doi.org/10.1590/S010288392001000300011.

Dubal S. The neoliberalization of football: rethinking neoliberalism through the commercialization of the beautiful game. Int Rev Sociol Sport 2010;45(2):123-46. http://dx.doi.org/10.1177/1012690210362426.

Edgar A. The beauty of sport. Sport Ethics Philos 2013;7(1):10020. http://dx.doi.org/10.1080/17511321.2013.761886.

Graça LG, Lacerda TO. Da estética do desporto à estética do futebol. Rev Bras Ciênc Esporte 2011;33(2):427-44. http:// dx.doi.org/10.1590/S0101-32892011000200010.

Gumbrecht HU. A forma da violência. Folha de São Paulo, São Paulo, 2001.

Hallinan C, Hughson J. The beautiful game in Howard's 'Brutopia': football, ethnicity and citizenship in Australia. Soccer Soc. 2009;10(1):1-8. http://dx.doi. org/10.1080/14660970802472627.

Hughes M, Franks I. Analysis of passing sequences, shots and goals in soccer. J Sports Sci 2005;23(5):509-14. http://dx.doi.org/10.1080/02640410410001716779. PMid:16194998.

Hughes MD, Bartlett RM. The use of performance indicators in performance analysis. J Sports Sci 2002;20(10):73954. http://dx.doi.org/10.1080/026404102320675602. PMid:12363292.

Jones PD, James N, Mellalieu SD. Possession as a performance indicator in soccer. Int J Perform Anal Sport 2004;4(1):98102. http://dx.doi.org/10.1080/24748668.2004.11868295.
Krippendorff K. Content analysis: an introduction to its methodology. 3rd ed. London: Sage; 2013.

Lacerda TO. Elementos para a construção de uma Estética do Desporto [dissertação]. Porto: Departamento de Estética do Desporto, Faculdade de Ciências do Desporto e Educação Física; 2002a.

Lacerda TO. A estética como uma dimensão de referência para o desporto do século XXI. Revista Horizonte 2002b;18(104):18-20.

Lago $C$. The influence of match location, quality of opposition, and match status on possession strategies in professional association football. J Sports Sci 2009;27(13):1463-9. http:// dx.doi.org/10.1080/02640410903131681. PMid:19757296.

Lago-Ballesteros J, Lago-Peñas C. Performance in team sports: identifying the keys to success in soccer. J Hum Kinet 2010;25:8591. http://dx.doi.org/10.2478/v10078-010-0035-0.

Lago-Peñas C, Dellal A. Ball possession strategies in elite soccer according to the evolution of the match-score: The influence of situational variables. J Hum Kinet 2010;25(1):93-100. http://dx.doi.org/10.2478/v10078010-0036-z.

Peterson RA, Merunka DR. Convenience samples of college students and research reproducibility. J Bus Res 2014;67(5):1035-41. http://dx.doi.org/10.1016/j. jbusres.2013.08.010.

Stolen T, Chamari K, Castagna C, Wisloff U. Physiology of soccer: an update. Sports Med 2005;35(6):501-36. http://dx.doi.org/10.2165/00007256-200535060-00004. PMid:15974635.

Taylor C. The beautiful game: a journey through Latin American football. London: Victor Gollancz Ltd.; 1998.

Vrooman J. Theory of the beautiful game: the unification of european football. Scott J Polit Econ 2007;54(3):314-54. http://dx.doi.org/10.1111/j.1467-9485.2007.00418.x. 